\title{
Investigating the Selectivity of Allosteric Inhibitors for Mutant T790M EGFR over Wild Type Using Molecular Dynamics and Binding Free Energy Calculations
}

\author{
Annachiara Tinivella and Giulio Rastelli*(i) \\ Department of Life Sciences, University of Modena and Reggio Emilia, Via Campi 103, 41125 Modena, Italy \\ Supporting Information
}

\begin{abstract}
The recent discovery of the fourth generation EAI045 allosteric inhibitor, which potently and selectively inhibits mutant EGFR, represents an important step forward for the treatment of non-small cell lung cancer. However, the structural determinants of EAI045 selectivity with respect to the wild type (wt) protein have not been fully investigated. To this aim, we performed a comparative analysis of long-scale molecular dynamics simulations and binding free energy calculations on wt and T790M EGFR in complexes with the EAI001 and EAI045 allosteric ligands. Unexpectedly, we found that the observed selectivity for T790M EGFR over wt is not due to more favorable interactions of the two ligands with the mutated gatekeeper residue, as previously suggested.

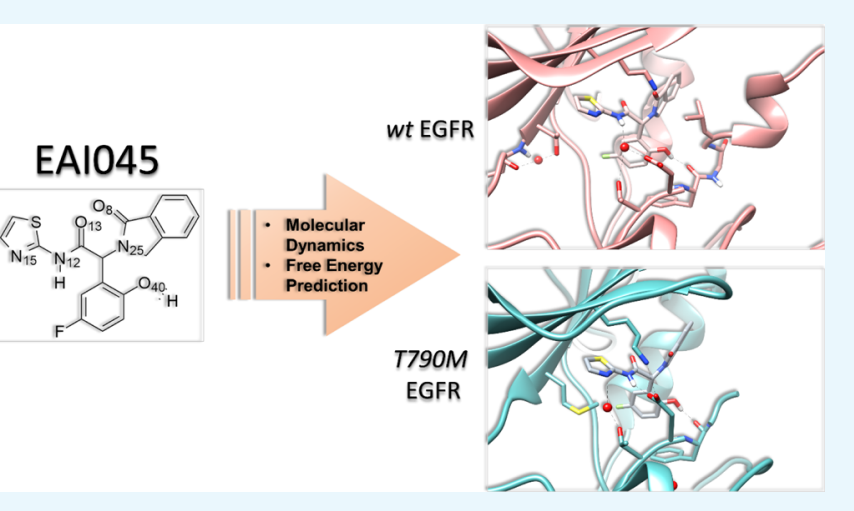
Rather, the allosteric ligands were engaged in a direct hydrogen bond with the Asp855 residue of the DFG motif in mutant T790M but not in wt, in which the hydrogen bond was found to be water-mediated. Per-residue decomposition of binding free energies suggests that the loss of a direct interaction with Asp855 is the main cause of inhibitor selectivity. Moreover, the possibility that the allosteric ligands and adenosine triphosphate may have synergistic binding effects, as previously observed in MEK allosteric inhibitors, was investigated. Altogether, the results suggest that ligand selectivity arises from direct hydrogen bonds with the Asp855 side chain, and that the design of mutant-selective inhibitors should be focused on ligands that form direct hydrogen bonds with Asp855 in T790M EGFR but not in wt EGFR. These results may provide useful hints for future structural design of mutant-selective allosteric inhibitors that spare wt EGFR, which is a highly desirable goal.
\end{abstract}

\section{INTRODUCTION}

Developing mutant-selective inhibitors of the tyrosine kinase epidermal growth factor receptor (EGFR) is of utmost importance. To date, six FDA-approved EGFR inhibitors are used in the clinic to treat non-small cell lung cancer (www. brimr.org/PKI/PKIs.htm; accessed on Nov 19, 2018). Unfortunately, the T790M secondary mutation of the gatekeeper residue is one of the main causes of drug resistance to first (e.g. gefitinib and erlotinib) and second generation (e.g. afatinib and dacomitinib) adenosine triphosphate (ATP)competitive EGFR inhibitors. ${ }^{1,2}$ Indeed, the gatekeeper mutation is able to enhance the affinity for the ATP substrate. ${ }^{3}$ Recently, the efforts potentially able to circumvent this problem were directed toward the identification of allosteric ligands, which bind to less conserved binding pockets and stabilize the inactive conformation of the receptor, whose dimerization is hampered by displacement of the regulatory $\alpha \mathrm{C}$-helix. ${ }^{4}$ Structure-based design of allosteric inhibitors is a challenging task because of the scarcity of structural information. ${ }^{5}$ In fact, the first crystal structure of an allosteric inhibitor in the complex with mutant EGFR was reported only in 2016 (PDB code: 5D41). ${ }^{6}$ The mutant-selective inhibitor EAI001 binds to an inactive conformation of T790M EGFR and is located in the allosteric pocket created by an outward shift of the $\alpha \mathrm{C}$ helix (see Figure S1). The crystallographic structure of the complex with the optimized derivative EAI045 was obtained in late 2018, and presents an additional mutation known to confer resistance to ATP-competitive irreversible inhibitors (C797S). ${ }^{7}$ Very recently, Kannan et al. reported a molecular dynamics investigation of EAI045 complexes with various mutant EGFRs, but the reasons for the observed selectivity with respect to the wild type (wt) EGFR protein

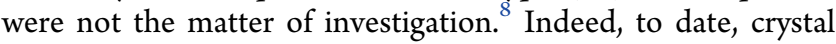
structures or computational models of the allosteric EAI compounds in the complex with wt EGFR have not been reported. EAI001 and its optimized derivative EAI045 proved to be selective inhibitors of mutant EGFR, ${ }^{6}$ but the selectivity determinants that discriminate against wt have not been specifically addressed.

In this study, we report the results of a comparative longscale $(2 \mu \mathrm{s})$ molecular dynamics and binding free energy prediction analysis performed on wt and T790M EGFR

Received: November 21, 2018

Accepted: November 23, 2018

Published: December 4, 2018 
complexes with the two allosteric ligands EAI001 and EAI045. Specifically, we propose and validate a binding mode of the EAI compounds on wt EGFR, then we compare it with the binding mode observed in the respective T790M EGFR mutant complexes. The results shed light on an interesting and far from obvious explanation to the observed selectivity, which points the attention to a water molecule that bridges the allosteric ligand to the DFG motif in wt, but not in T790M EGFR. Moreover, we addressed the questions as to why EAI045 is more potent than EAI001 and the possibility that the allosteric ligands and ATP may have synergistic binding effects, as previously observed in MEK allosteric inhibitors. ${ }^{9,10}$

\section{RESULTS AND DISCUSSION}

Generation of EGFR/EAI Complexes. The crystal structure of EAI001 in the complex with T790M EGFR (PDB code: 5D41, chain A) ${ }^{6}$ was used to perform molecular dynamics simulations of the T790M EGFR/EAI001/ATP complex (Figure S1). As for the complex with the optimized inhibitor EAI045, the starting complex was generated by docking EAI045 to the 5D41 structure (see Methods), so that all further analyses could be performed on the same protein structure. Remarkably, the superimposed docked pose resulted quite similar to the crystallographic pose of EAI045 in the complex with C797S/T790M EGFR reported later (PDB code $5 \mathrm{ZWJ}){ }^{7}$ with a root mean square deviation (RMSD) of only $0.81 \AA$, despite the absence of $\mathrm{Mg}$-ATP and the presence of an additional mutation (C797S) in this crystal structure. To be consistent with the 5D41 structure, we generated a ternary T790M EGFR/EAI045/ATP complex, whereas the recently released structure was crystallized without ATP. Regarding the generation of wt-EGFR/EAI complexes, we were faced with the notion, as reported in the article by Jia et al., that the binding mode of EAI001 seems to be incompatible with the structure of wt EGFR. ${ }^{6}$ In fact, in the structure analyzed by the authors (PDB code: 2GS7), and lately also by Kannan and coworkers, ${ }^{8}$ the allosteric pocket is partially occluded by a short helix at the beginning of the activation loop, which is typical of kinases in the inactive conformation. We recently reported a comprehensive analysis and comparison of the structural kinome, which explored the different geometrical and physicochemical properties of the allosteric pocket by taking the T790M EGFR crystal structure of 5D41 as a reference. ${ }^{11}$ Based on this analysis, the PDB structure of wt EGFR in the complex with a 4-amino-6-arylamino-pyrimidine-5-carbaldehyde hydrazine derivative (PDB code 2RGP) had an allosteric pocket geometrically similar to that of 5D41. Interestingly, this structure was crystallized with an ATP-competitive ligand possessing a chemical moiety that extended into the allosteric pocket, thus resulting in a more open allosteric pocket. ${ }^{12}$ With respect to 2GS7, residues Leu858 and Leu861 of this structure are slightly shifted, thus allowing the EAI inhibitors to dock in the allosteric pocket with minor adjustment of nearby residue side chains. In fact, by means of induced fit docking, we were able to obtain wt EGFR/EAI complexes that closely matched the crystallographic poses observed in mutant EGFR.

Molecular Dynamics of EAI001 and EAI045 Complexes with wt and T790M EGFR. Each ternary EGFR/ EAI/ATP complex was simulated for over $0.5 \mu \mathrm{s}$ in water (periodic boundary conditions), resulting in a total of $2 \mu \mathrm{s}$ simulation time. The binding modes of the allosteric inhibitors and ATP were conserved throughout the entire MD simulation of all four complexes, as evidenced by the small RMSD's of ligand positions reported in Table 1. Likewise, an inspection of the $\mathrm{C} \alpha$ carbon deviations of the residues composing the allosteric pocket showed no considerable deviations from the original position.

Table 1. RMSD $(\AA)$ of Protein C $\alpha$ Carbons and of the Ligand and ATP Heavy Atoms for Each EGFR/EAI/ATP Molecular Dynamics Simulation

\begin{tabular}{|c|c|c|c|c|}
\hline & $\pi^{s}$ & 11 & $\begin{array}{l}\frac{\text { EAl001 }}{R_{1}=R_{2}=H} \\
\text { EAI045 } \\
R_{1}=O H \\
R_{2}=F\end{array}$ & \\
\hline & \multicolumn{2}{|l|}{ EAI001 } & \multicolumn{2}{|l|}{ EAI045 } \\
\hline & wt & $\mathrm{T} 790 \mathrm{M}$ & wt & T790M \\
\hline Protein $\mathrm{C} \alpha$ & $2.0 \pm 0.2$ & $2.1 \pm 0.4$ & $1.9 \pm 0.2$ & $2.2 \pm 0.3$ \\
\hline Ligand & $1.2 \pm 0.2$ & $1.0 \pm 0.2$ & $1.5 \pm 0.2$ & $0.9 \pm 0.2$ \\
\hline ATP & $1.3 \pm 0.2$ & $1.4 \pm 0.2$ & $1.3 \pm 0.4$ & $1.5 \pm 0.3$ \\
\hline
\end{tabular}

The generated MD trajectories were then used to carry out an analysis of the protein-ligand hydrogen bond networks, including water-mediated bridge interactions (Table 2). The analysis of the T790M EGFR/EAI001 MD trajectory confirmed that the interactions observed in the 5D41 crystal structure are indeed conserved throughout the MD simulation, for example the hydrogen bond between the amide nitrogen N12 and the side chain of Asp855 (Table 2). The hydrogen bond between Lys745 and the 08 carbonyl of the isoindolinone ring was not present in the $\mathrm{MD}$ simulation, this residue being preferably engaged in electrostatic interactions with one of the phosphate oxygens of ATP. The binding mode of EAI045 closely overlapped that of EAI001, the additional hydroxyl group forming a hydrogen bond with the backbone carbonyl of the Phe 856 residue of the DFG motif (Table 2 and Figure 1A). This hydrogen bond is maintained throughout the entire simulation. Apart from this additional hydrogen bond and the presence of the fluorine, our simulations did not point out additional interactions that may explain the higher inhibitory activity of EAI045 with respect to EAI001. ${ }^{6}$

The MD simulations of the two wt EGFR/EAI/ATP complexes were run using the same protocol described for mutant EGFR complexes. Again, the average RMSD for ligand position and protein $\mathrm{C} \alpha$ carbons was relatively small (Table 1 ). However, a closer inspection of the hydrogen bond network resulting from these MD simulations revealed that the hydrogen bond between the amide nitrogen (N12) and the Asp855 side chain was not present in the wt simulations (Table 2). This behavior was observed for both EAI001 and EAI045. Unexpectedly, the amide nitrogen and the Asp855 side chain were bridged by one water molecule, instead of a direct hydrogen bond. Moreover, the salt bridge between Lys745 and Asp855 was lost and never recovered, the Lys745 residue interacting with the $\mathrm{O} 8$ carbonyl of the ligand, and the Asp855 side chain interacting with the Thr854 residue ahead of the DFG (Figure 1B). Remarkably, this interaction pattern resulting from $\mathrm{MD}$ was not present neither in the mutant EGFR crystal structures nor in the initial docked complexes. Therefore, the results obtained in wt EGFR point the attention 
Table 2. Percentage of the Occurrence of Ligand-EGFR Hydrogen Bonds Sampled by MDs ${ }^{a}$

\begin{tabular}{|c|c|c|c|c|}
\hline & & EAI001 & & EAI045 \\
\hline & wt & T790M & wt & T790M \\
\hline \multicolumn{5}{|l|}{ HYDROGEN BONDS } \\
\hline K745 - LIGAND (08) & $86.5 \%$ & $0.4 \%$ & $90.1 \%$ & $0.0 \%$ \\
\hline K745 - LIGAND (N15) & $0.0 \%$ & $7.4 \%$ & $0.0 \%$ & $9.2 \%$ \\
\hline K745 - LIGAND (N12) & $0.0 \%$ & $4.6 \%$ & $0.0 \%$ & $4.7 \%$ \\
\hline D855 - LIGAND (N12) & $0.0 \%$ & $99.5 \%$ & $2.5 \%$ & $99.7 \%$ \\
\hline F856 (backbone) - LIGAND (040) & / & l & $92.1 \%$ & $98.2 \%$ \\
\hline K745 - ATP (02A) & $0.1 \%$ & $93.8 \%$ & $2.5 \%$ & $96.5 \%$ \\
\hline |T854 - D855 (backbone) & $22.2 \%$ & $10.0 \%$ & $18.7 \%$ & $7.6 \%$ \\
\hline T854 - D855 (side-chain) & $7.1 \%$ & $3.0 \%$ & $2.8 \%$ & $0.1 \%$ \\
\hline \multicolumn{5}{|c|}{ WATER MEDIATED HYDROGEN BONDS } \\
\hline K745 - W - LIGAND (08) & $5.1 \%$ & $33.3 \%$ & $7.9 \%$ & $39.8 \%$ \\
\hline T854 - W - LIGAND (N12) & $62.70 \%$ & $22.30 \%$ & $76.20 \%$ & $14.50 \%$ \\
\hline T854 - W - LIGAND (N15) & $45.8 \%$ & $23.1 \%$ & $59.1 \%$ & $44.5 \%$ \\
\hline D855 - W - LIGAND (N12) & $94.4 \%$ & $35.9 \%$ & $100.0 \%$ & $29.3 \%$ \\
\hline G857 - W - LIGAND (040) & / & $14.0 \%$ & / & $0.8 \%$ \\
\hline ATP - W - LIGAND (N15) & $62.0 \%$ & $23.1 \%$ & $67.9 \%$ & $46.1 \%$ \\
\hline T790 - W - Q791 & $56.2 \%$ & l & $56.6 \%$ & / \\
\hline T790 -W - T854 & $0.0 \%$ & I & $0.2 \%$ & I \\
\hline T790 - W - ATP & $0.0 \%$ & l & $6.7 \%$ & / \\
\hline K745 - W - ATP & $11.7 \%$ & $51.7 \%$ & $9.1 \%$ & $34.9 \%$ \\
\hline K745 - W - D855 & $64.4 \%$ & $0.4 \%$ & $60.4 \%$ & $0.3 \%$ \\
\hline T854 - W - ATP & $22.2 \%$ & $6.2 \%$ & $21.4 \%$ & $6.6 \%$ \\
\hline T854 - W - D855 & $1.0 \%$ & $15.0 \%$ & $1.8 \%$ & $17.7 \%$ \\
\hline D855 - W - ATP & $87.7 \%$ & $2.5 \%$ & $87.6 \%$ & $1.8 \%$ \\
\hline G857 - W - D855 & $0.0 \%$ & $14.9 \%$ & $0.0 \%$ & $3.0 \%$ \\
\hline \multicolumn{5}{|l|}{ IONIC INTERACTIONS } \\
\hline K745 - D855 & $0.1 \%$ & $98.2 \%$ & $2.5 \%$ & $98.0 \%$ \\
\hline
\end{tabular}

${ }^{a} \mathrm{~A}$ "/" indicates that the protein does not possess the required group to form the interaction.

A)

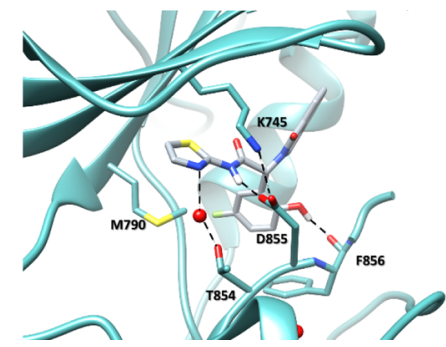

B)

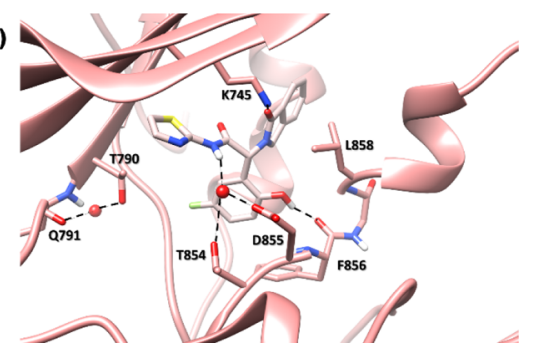

Figure 1. (A) Representative structure of the T790M EGFR/EAI045/ATP complex obtained by MD. (B) Representative structure of the wt EGFR/EAI045/ATP complex obtained by MD. Water molecules are shown as red dots. Hydrogen bonds are highlighted with black dotted lines. The ATP molecule was omitted for clarity.

to the absence of a direct interaction between the ligand and the Asp855 side chain, which clearly distinguishes these complexes from those obtained in mutant EGFR (Table 2).
The ligands are still held in place by the additional interaction between the carbonyl oxygen $\mathrm{O} 8$ and the Lys745, but the direct interaction with Asp855 is lost. The additional hydroxyl 
Table 3. MM-PBSA Binding Energies $(\mathrm{kcal} / \mathrm{mol})$ of EAI001 and EAI045 to wt and T790M EGFR ${ }^{a}$

\begin{tabular}{|c|c|c|c|c|}
\hline \multirow[b]{2}{*}{$(\mathrm{kcal} / \mathrm{mol})$} & \multicolumn{2}{|c|}{ EAI001 } & \multicolumn{2}{|c|}{ EAI045 } \\
\hline & wt & T790M & wt & $\mathrm{T} 790 \mathrm{M}$ \\
\hline ternary complex (EGFR/ligand/ATP) including ATP contribution & $-29.0 \pm 3.9$ & $-36.0 \pm 3.5$ & $-30.9 \pm 4.3$ & $-37.7 \pm 3.8$ \\
\hline ternary complex (EGFR/ligand/ATP) excluding ATP contribution & & & $-32.1 \pm 3.9$ & $-37.6 \pm 3.4$ \\
\hline binary complex (EGFR/ligand) & & & $-39.3 \pm 4.6$ & $-41.1 \pm 4.3$ \\
\hline
\end{tabular}

${ }^{a}$ Energies were calculated for the ternary complexes (EGFR/ligand/ATP), EGFR/ligand/ATP ternary complexes by excluding the ATP contribution, and binary complexes obtained by MD simulations of EGFR/ligand complexes (i.e. no ATP in the trajectory).

group of EAI045 hydrogen bonded with the backbone carbonyl of Phe856 for the whole duration of the MD simulation, as already observed in the MD simulations of mutant EGFR complexes.

To conclude, the MD simulations suggest that the allosteric ligands EAI001 and EAI045 form direct hydrogen bonds with the Asp855 side chain in mutant EGFR, but not in the wt protein.

Selectivity Determinants from Binding Free Energy Calculations and Per-Residue Free Energy Decomposition. To further investigate the origin of the selectivity of EAI ligands for T790M EGFR, we also calculated the binding free energies of the two compounds to the respective wt and mutant proteins. Remarkably, we found that the free energies of binding of EAI001 and EAI045 to T790M EGFR are around $7 \mathrm{kcal} / \mathrm{mol}$ more favorable with respect to those for wt EGFR (Table 3). This result is in agreement with the experimentally observed selectivity of EAI compounds for mutant EGFR (EAI045's inhibitory activity being 32-fold better for T790M EGFR over the wt). ${ }^{6}$ Although absolute free energies calculated with MM-PBSA are known not to be quantitatively comparable with experimental binding free energies calculated from $K_{\mathrm{i}}$ or $\mathrm{IC}_{50}$ values, their relative variations are indeed relevant and may be qualitatively used for comparative purposes. $^{13,14}$ Free energy predictions also suggest that the binding free energy of EAI045 is around $2 \mathrm{kcal} / \mathrm{mol}$ more favorable than that of EAI001 in both the wt and T790M EGFR simulations (Table 3), again in agreement with experimental data.

Finally, to evaluate which amino acid residues contributed the most to the variations of total binding free energies reported in Table 3, the calculated total free energies were decomposed into their per-residue contributions (see Methods). Figure 2 shows the average contribution of residues with $0.2<\Delta G_{\text {MM-PBSA }}<-0.2 \mathrm{kcal} / \mathrm{mol}$ values. Interestingly, although Jia et al. proposed that EAI selectivity for mutant EGFR may be because of favorable interactions of the sulfur of the thiazole ring with the mutated Met790 side chain, we found that the relative contribution of the methionine to the binding free energy was only $0.5 \mathrm{kcal} / \mathrm{mol}$ more favorable with respect to the contribution of the threonine residue of the wt (Figure 2A). On the contrary, the Asp855 residue contributed with a much more significant $(3.5 \mathrm{kcal} / \mathrm{mol})$ stabilization to the binding free energy of T790M complexes as opposed to wt (Figure 2A). This finding is in agreement with our observation that EAI compounds do not form a direct hydrogen bond with Asp855 in wt EGFR. Therefore, structures and free energies agree in saying that most of the selectivity comes from the lack of a direct hydrogen bond with Asp855 in wt EGFR. Indeed, while Asp855 has a positive (i.e. unfavorable) $\Delta G_{\text {MM-PBSA }}$ contribution in wt (Figure 2A), the contribution becomes significantly negative (i.e. favorable) in mutant EGFR. Figure $2 \mathrm{~A}$ also shows that Lys745 has a slightly more favorable
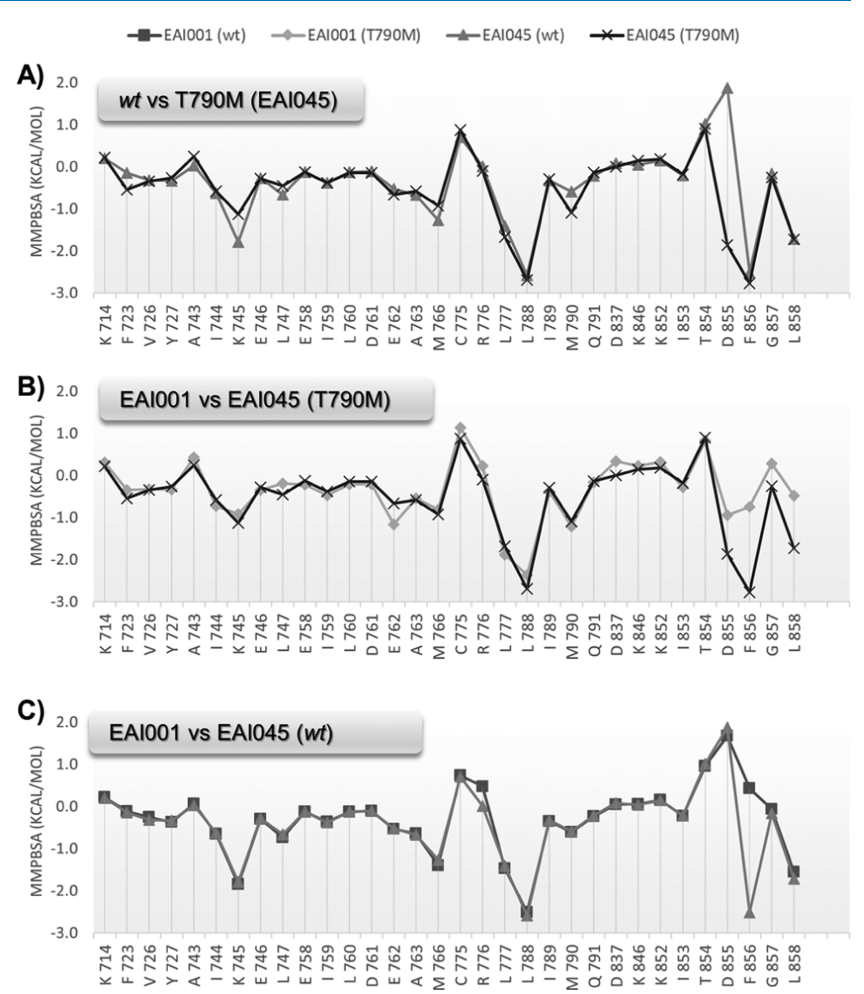

Figure 2. Binding free energy decomposition profiles (MM-PBSA) of (A) wt vs T790M EGFR/EAI045/ATP MD simulations; (B) T790M EGFR/EAI001 vs EAI045/ATP MD simulations; (C) wt EGFR/ EAI001 vs EAI045/ATP MD simulations. Only residues with $0.2<$ $\Delta G_{\text {MM-PBSA }}<-0.2 \mathrm{kcal} / \mathrm{mol}$ values are shown.

contribution in the wt-EGFR/EAI045 complex. This is in agreement with the detected hydrogen bond between the carbonyl oxygen $\mathrm{O} 8$ and the residue side chain. Interestingly, this additional interaction could not compensate for the loss of the EAI045-Asp855 hydrogen bond. Finally, Figure 2B compares the residue contributions to the binding free energy of EAI001 and EAI045 to T790M EGFR.

As expected, most of the additional stabilization observed for EAI045 complexes comes from the DFG motif, and in particular from the Phe 856 residue that provides additional 3 $\mathrm{kcal} / \mathrm{mol}$ stabilization to the overall binding free energy with respect to EAI001 (Figure 2B). This finding nicely correlates with the establishment of an additional hydrogen bond via the hydroxyl group. Asp855, Gly857, and Leu858 also showed more negative (i.e. favorable) contributions of around $1 \mathrm{kcal} /$ mol each. It is likely that the additional hydrogen bond with the Phe 856 carbonyl anchors the molecule more tightly to the DFG motif, resulting in globally stronger interactions. Indeed, Figure 2C, which compares the residue contributions of EAI001 and EAI045 complexes with wt EGFR, shows that the only difference comes from Phe856. Asp855, Gly857 and 

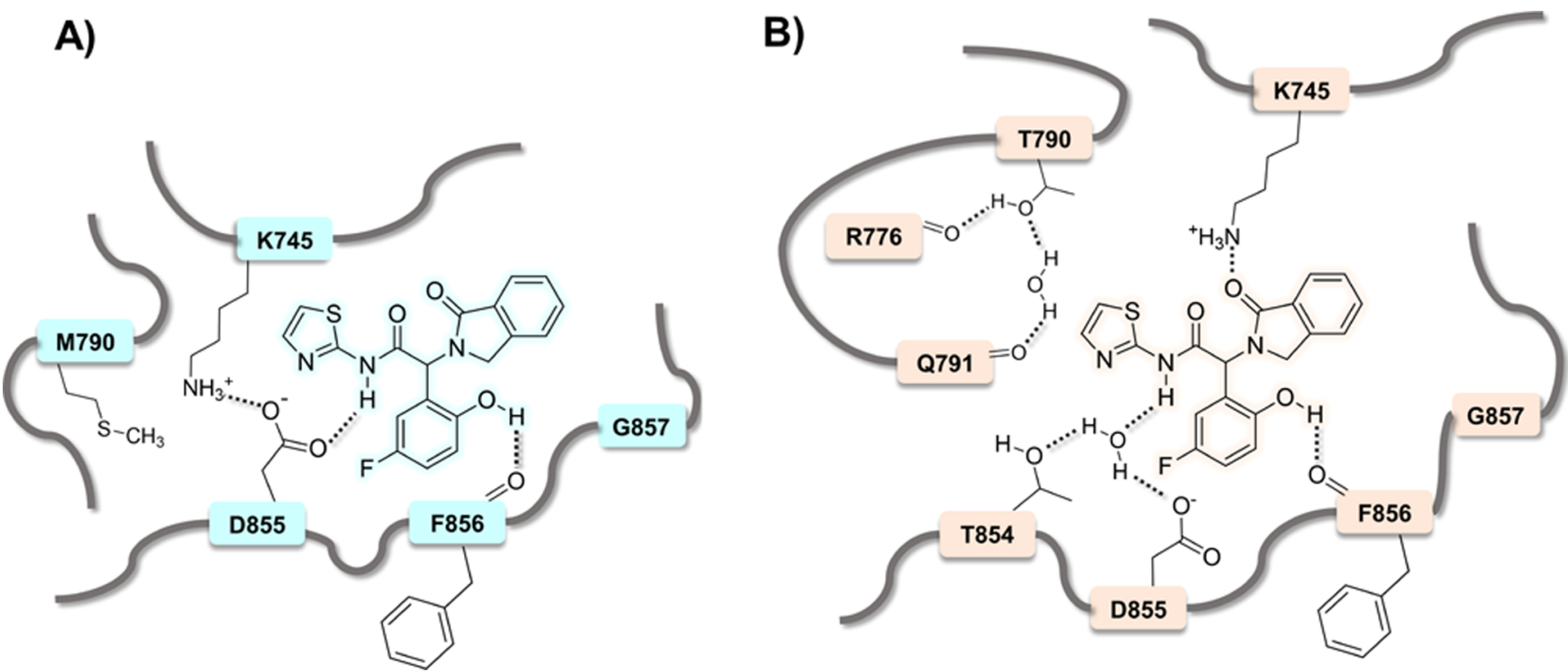

Figure 3. 2D representation of the binding mode of EAI045 to T790M EGFR (panel A) and wt EGFR (panel B), according to MD simulations. Relevant explicit waters and hydrogen bonds are showed. The ATP molecule was omitted for clarity.

Leu858 do not appear to be affected by the additional hydrogen bond with the hydroxyl group, in agreement with the fact that none of them forms a direct hydrogen bond with the ligand.

Testing a Possible Synergistic Effect of ATP on the Binding of EAI045. Finally, we investigated whether ATP could provide synergistic binding effects with the EAI compounds, as already observed for other type III allosteric ligands of MEKs. ${ }^{9,10}$ Although the 5D41 PDB structure was crystallized with both EAI001 and the ATP derivative AMPPNP (phosphoaminophosphonic acid-adenylate ester), ${ }^{6}$ the more recent crystal structure of T790M/C797S EGFR in the complex with EAI045 was obtained without ATP (5ZWJ). Moreover, our T790M EGFR MD simulations identified direct interactions between residue Lys745 and ATP, as well as water-mediated interactions between Thr854, Asp855, and ATP in wt EGFR MD simulations.

Therefore, we repeated the binding free energy calculations on the ternary EGFR/EAI045/ATP MD complexes by excluding ATP from the list of residues contributing to the overall binding free energy. By excluding the contribution of ATP, binding free energies were only $1 \mathrm{kcal} / \mathrm{mol}$ more negative in wt EGFR and almost identical in T790M EGFR (Table 3). Therefore, our results suggest that ATP does not provide synergistic effects with EAI compounds. Binding free energies were also calculated by performing additional MD simulations on the binary EGFR/EAI045 complexes, that is, by generating new $0.25 \mu \mathrm{s}$ MD trajectories without bound ATP (see Methods). In the binary complexes, binding free energies of EAI045 were around 8 and $3 \mathrm{kcal} / \mathrm{mol}$ more favorable than those calculated in the ternary complexes (Table 3), providing further evidence of a lack of synergistic effects. This difference is mainly due to the establishment of a hydrogen bond between the $\mathrm{O} 8$ carbonyl of EAI045 and the Lys745 side chain (see Supporting Information), which does not have to compete with the Lys745/ATP ionic interaction observed in the ternary complexes. This is in agreement with what is proposed by Zhao et al., who suggested that without the ATP molecule Lys745 shifts toward the carbonyl O8 of the EAI045 compound.

\section{CONCLUSIONS}

Mutant-selective inhibitors binding to EGFR with an allosteric mechanism have been recently described. Despite being selective for mutant T790M EGFR, a structural explanation of such biological behavior is still missing.

In an effort to identify structural motifs explaining the different affinity of EAI001 and EAI045 allosteric inhibitors for the wt and mutant EGFR proteins, molecular dynamics and binding free energy calculations were performed. To this aim, $0.5 \mu \mathrm{s}$ MD simulations of the two EAI compounds in complex with wt and T790M EGFR proteins were performed. Then, the obtained trajectories were analyzed in order to investigate and compare the resulting hydrogen bond networks. Results from these analyses showed that the Asp855 residue plays a key role in ligand selectivity. In fact, while this residue establishes direct hydrogen bonds with the EAI compounds in both the X-ray and MD-generated T790M-EGFR structures, this interaction becomes water-mediated in wt EGFR, a water molecule bridging the amino group of the EAI ligands to the Asp855 side chain. The different hydrogen bonding patterns are graphically illustrated in Figure 3 panel A (T790M) and panel $B$ (wt). Binding free energy calculations with the MM-PBSA methods clearly indicated that the compounds bind preferentially to T790M EGFR and that EAI045 binds more tightly than EAI001, in agreement with the experiment. Per-residue decomposition of binding free energies clearly pointed to the discriminating role of Asp855, in agreement with the observed structural differences arising from the MD simulations.

In conclusion, we propose that ligand selectivity arises from direct hydrogen bonding of the ligand with the Asp855 side chain in mutant EGFR but not in wt, in which the interaction is water-mediated and much weaker. Therefore, the design of mutant-selective inhibitors should be focused on ligands that form direct hydrogen bonds with Asp855 in T790M EGFR but not in wt EGFR structures, as exemplified in Figure 3 panel A (T790M) and panel B (wt). The different hydrogen bonding patterns depicted in the two panels may help to devise ad hoc structure-based pharmacophores or docking screening experiments to assist the design of mutant-selective ligands. It will be interesting to see if future experiments will confirm our 
predictions. Meanwhile, our results may provide useful hints for the discovery of mutant-selective allosteric ligands of EGFR, which is a highly desirable goal.

\section{MATERIALS AND METHODS}

Structure Preparation. Crystal structures were downloaded from the Protein Data Bank and processed with the Schrödinger Suite 2014-3 Protein Preparation Wizard (PPW) tool. PPW automatically adjusted the ionization and tautomerization state of the protein at a neutral $\mathrm{pH}$ (PROPKA was used to predict the $\mathrm{pKa}$ of residues at $\mathrm{pH}=7)$, set the orientation of any misoriented groups (Asn, Gln, and His residues), and optimized the hydrogen bond network. All residues were found to be in standard ionization states. Chain $\mathrm{B}$ of the PDB structure 5D41 was deleted, as it is not bound to the EAI ligand. Uncapped amino acidic $\mathrm{N}$ and $\mathrm{C}$ terminals were treated with the "cap termini" option. The choice of the crystal structures of wt EGFR to be used for docking was based on our recent work that compared the geometrical and physico-chemical features of the allosteric pocket in the structural kinome. ${ }^{11}$ This identified 2RGP as a high-resolution crystal structure of wt EGFR, having a close match of the allosteric pocket with that of 5D41. The PDB structure of wt 2RGP was superimposed to that of 5D41, and the EAI001 ligand was added to the allosteric pocket. In a final step, the ligand-protein complexes were refined to relieve steric clashes performing a restrained minimization on the proteins' atoms. The OPLS 2005 force field was used, and the minimization was terminated when a final RMSD of $0.30 \AA$ with respect to the input protein coordinates was reached. The $3 \mathrm{D}$ structures of ligands EAI001 and EAI045 were drawn with Maestro, and prepared with the LigPrep module. Induced Fit Docking (IFD) was used to simulate the binding modes of the EAI compounds in the wt and T790M mutant proteins. ${ }^{15}$ IFD was performed with standard settings except for an additional trimming of the Leu858 and Met766 side chains. A maximum number of 20 poses was saved for each ligand and submitted to the ensuing prediction of side-chain orientation of residues within $5 \AA$ around the ligand, as calculated by Prime. After the Prime minimization, a Glide SP redocking of each protein-ligand complex structure was performed. Finally, the binding energy (IFDScore) for each output pose was calculated to score the resulting complexes. Resulting poses were visually analyzed and compared with the corresponding initial crystal structures.

Molecular Dynamics Protocol. The T790M EGFR/ EAI001/ATP MD simulation was performed starting from the crystal structure of 5D41, whereas the starting point for the other simulations were the top-scoring complexes obtained by IFD, as described above. Atomic charges of the ligand were computed with antechamber (BCC charge method). The corresponding protein was saved in the PDB format, deleting all hydrogen atoms. The topologies were created with the program tleap of the Amber14 suite. Parameters from the Manchester University AMBER parameter database (http:// research.bmh.manchester.ac.uk/bryce/amber/) were used for $\mathrm{ATP}^{16}$ and $\mathrm{Mg}$ ion. ${ }^{17}$ The protein-ligand complex was enclosed in a truncated octahedral TIP3P water box with a radius of $10 \AA$. Counter ions were added to neutralize the complexes; $5 \mathrm{Na}^{+}$ions were required to neutralize T790M EGFR (5D41) whereas $3 \mathrm{Cl}^{-}$ions were added to neutralize the wt EGFR (2RGP) complexes. For all the simulations, the coordinates of the phosphoaminophosphonic acid-adenylate ester (AMP-PNP, PDB ID: ANP) and Mg ion were obtained from the 5D41 PDB structure (chain A). The nitrogen atom of AMP-MNP was edited to an atom of oxygen to obtain the ATP structure. All molecular dynamics runs were performed using Amber 14. ${ }^{18}$ An initial minimization of the solvated complexes was carried out on the whole system. The minimization consisted of 1000 steps of steepest descent minimization followed by 1000 steps of the conjugate gradient minimization. The minimized system was heated to $300 \mathrm{~K}$ with a constant volume $0.5 \mathrm{~ns}$ simulation, whereas the protein $\mathrm{C} \alpha$ carbons were held fixed with a $10 \mathrm{kcal} / \mathrm{mol}$ constraint. Subsequently, the system was re-equilibrated with a 3 ns constant pressure MD to allow adjustment of the density of the system. The pressure was set to 1 atm with isotropic position scaling (ntp = 1). Finally, the constraints on the $\mathrm{C} \alpha$ carbons were released, and a MD production run without constraints at 1 atm constant pressure was calculated. The total simulation time for each complex was set to $0.5 \mu \mathrm{s}$. A time step of 2 fs was set, and the SHAKE algorithm was used to constrain bonds involving hydrogen atoms. Analysis of the resulting trajectories was carried out by means of calculation of rmsd, hydrogen bond network, and water occupancy using the program cpptraj of the Amber14 suite (using the rms, hbond and watershell commands, respectively).

MM-PBSA Calculations. Binding free energy calculations were performed by means of the MMPBSA.py module of the Amber14 suite. Prior to the calculations, counter ions were removed from the MD trajectory coordinate file. New prmtop files were calculated using cpptraj for the solvated and unsolvated complexes and for the isolated receptor and ligand structures. MM-PBSA parameters were set as in previous investigations. ${ }^{13} \mathrm{~A}$ total of three free energy calculations were performed: (i) on the ternary EGFR/EAI045/ATP complexes; (ii) an additional calculation on the same trajectory, but excluding the ATP contribution by extracting the ATP molecule from the trajectory; (iii) on the binary EGFR/ EAI045 complexes MD. Moreover, binding free energies were decomposed based on their per-residue contributions using the MMPBSA.py module (idecomp = 1).

\section{ASSOCIATED CONTENT}

\section{S Supporting Information}

The Supporting Information is available free of charge on the ACS Publications website at DOI: 10.1021/acsomega.8b03256.

Supporting Information contains a representation of the EGFR binding site of 5D41, average rmsd values, and percentages of occurrence hydrogen bond for the binary $\mathrm{MD}$ simulations (EGFR/EAI) (PDF)

\section{AUTHOR INFORMATION}

\section{Corresponding Author}

*E-mail: giulio.rastelli@unimore.it.

ORCID

Giulio Rastelli: 0000-0002-2474-0607

\section{Author Contributions}

The manuscript was written through contributions of all the authors. All the authors have given approval to the final version of the manuscript. Conceiving the project (G.R.), molecular modeling and analysis of results (A.T.), manuscript preparation (A.T. and G.R.). 


\section{Funding}

This work was supported by a grant from the Associazione Italiana per la Ricerca sul Cancro (AIRC IG 15993)

\section{Notes}

The authors declare no competing financial interest.

\section{ABBREVIATIONS}

EGFR, epidermal growth factor receptor; PDB, Protein Data Bank; PPW, Protein Preparation Wizard; IFD, Induced Fit Docking; wt, wild type; ATP, adenosine triphosphate; AMPPNP, 5'-adenylyl-imidodiphosphate

\section{REFERENCES}

(1) Nguyen, K.-S. H.; Kobayashi, S.; Costa, D. B. Acquired Resistance to Epidermal Growth Factor Receptor Tyrosine Kinase Inhibitors in Non-Small-Cell Lung Cancers Dependent on the Epidermal Growth Factor Receptor Pathway. Clin. Lung Cancer 2009, 10, 281-289.

(2) Gazdar, A. F. Activating and Resistance Mutations of EGFR in Non-Small-Cell Lung Cancer: Role in Clinical Response to EGFR Tyrosine Kinase Inhibitors. Oncogene 2009, 28, S24-S31.

(3) Yun, C.-H.; Mengwasser, K. E.; Toms, A. V.; Woo, M. S.; Greulich, H.; Wong, K.-K.; Meyerson, M.; Eck, M. J. The T790M Mutation in EGFR Kinase Causes Drug Resistance by Increasing the Affinity for ATP. Proc. Natl. Acad. Sci. U.S.A. 2008, 105, 2070-2075.

(4) Palmieri, L.; Rastelli, G. $\alpha \mathrm{C}$ helix displacement as a general approach for allosteric modulation of protein kinases. Drug Discovery Today 2013, 18, 407-414.

(5) Liu, Y.; Gray, N. S. Rational Design of Inhibitors That Bind to Inactive Kinase Conformations. Nat. Chem. Biol. 2006, 2, 358-364.

(6) Jia, Y.; Yun, C.-H.; Park, E.; Ercan, D.; Manuia, M.; Juarez, J.; $\mathrm{Xu}$, C.; Rhee, K.; Chen, T.; Zhang, H.; et al. Overcoming EGFR(T790M) and EGFR(C797S) Resistance with Mutant-Selective Allosteric Inhibitors. Nature 2016, 534, 129-132.

(7) Zhao, P.; Yao, M.-Y.; Zhu, S.-J.; Chen, J.-Y.; Yun, C.-H. Crystal Structure of EGFR T790M/C797S/V948R in Complex with EAI045. Biochem. Biophys. Res. Commun. 2018, 502, 332-337.

(8) Kannan, S.; Venkatachalam, G.; Lim, H. H.; Surana, U.; Verma, C. Conformational Landscape of the Epidermal Growth Factor Receptor Kinase Reveals a Mutant Specific Allosteric Pocket. Chem. Sci. 2018, 9, 5212-5222.

(9) Fischmann, T. O.; Smith, C. K.; Mayhood, T. W.; Myers, J. E.; Reichert, P.; Mannarino, A.; Carr, D.; Zhu, H.; Wong, J.; Yang, R.-S.; Le, H. V.; Madison, V. S. Crystal Structures of MEK1 Binary and Ternary Complexes with Nucleotides and Inhibitors. Biochemistry 2009, 48, 2661-2674.

(10) Smith, C. K.; Windsor, W. T. Thermodynamics of Nucleotide and Non-ATP-Competitive Inhibitor Binding to MEK1 by Circular Dichroism and Isothermal Titration Calorimetry. Biochemistry 2007, $46,1358-1367$.

(11) Sturm, N.; Tinivella, A.; Rastelli, G. Exploration and Comparison of the Geometrical and Physicochemical Properties of an $\alpha \mathrm{C}$ Allosteric Pocket in the Structural Kinome. J. Chem. Inf. Model. 2018, 58, 1094-1103.

(12) Xu, G.; Abad, M. C.; Connolly, P. J.; Neeper, M. P.; Struble, G. T.; Springer, B. A.; Emanuel, S. L.; Pandey, N.; Gruninger, R. H.; Adams, M.; Moreno-Mazza, S.; Fuentes-Pesquera, A. R.; Middleton, S. A. 4-Amino-6-Arylamino-Pyrimidine-5-Carbaldehyde Hydrazones as Potent ErbB-2/EGFR Dual Kinase Inhibitors. Bioorg. Med. Chem. Lett. 2008, 18, 4615-4619.

(13) Rastelli, G.; Del Rio, A.; Degliesposti, G.; Sgobba, M. Fast and Accurate Predictions of Binding Free Energies Using MM-PBSA and MM-GBSA. J. Comput. Chem. 2010, 31, 797-810.

(14) Parenti, M. D.; Rastelli, G. Advances and Applications of Binding Affinity Prediction Methods in Drug Discovery. Biotechnol. Adv. 2012, 30, 244-250.
(15) Sherman, W.; Day, T.; Jacobson, M. P.; Friesner, R. A.; Farid, R. Novel Procedure for Modeling Ligand/Receptor Induced Fit Effects. J. Med. Chem. 2006, 49, 534-553.

(16) Meagher, K. L.; Redman, L. T.; Carlson, H. A. Development of Polyphosphate Parameters for Use with the AMBER Force Field. J. Comput. Chem. 2003, 24, 1016-1025.

(17) Allnér, O.; Nilsson, L.; Villa, A. Magnesium Ion-Water Coordination and Exchange in Biomolecular Simulations. J. Chem. Theory Comput. 2012, 8, 1493-1502.

(18) Case, D. A.; Babin, J.; Berryman, J. T.; Betz, R. M.; Cai, Q.; Cerutti, D. S.; Cheatham, T. E., III; Darden, T. A.; Duke, R. E.; Gohlke, H.; Goetz, A. W.; Gusarov, S.; Homeyer, N.; Janowski, P.; Kaus, J.; Kolossváry, I.; Kovalenko, A.; Lee, T. S.; LeGrand, S.; Luchko, T.; Luo, R.; Madej, B.; Merz, K. M.; Paesani, F.; Roe, D. R.; Roitberg, A.; Sagui, C.; Salomon-Ferrer, R.; Seabra, G.; Simmerling, C. L.; Smith, W.; Swails, J.; Walker, R. C.; Wang, J.; Wolf, R. M.; Wu, X.; Kollman, P. A. AMBER 14; University of California: San Francisco, 2014. 